\title{
Integration of Pediatric Hodgkin Lymphoma Treatment and Late Effects Guidelines: Seeing the Forest Beyond the Trees
}

\author{
Matthew J. Ehrhardt, MD, $\mathrm{MS}^{1,2}$; Jamie E. Flerlage, MD, $\mathrm{MS}^{1}$; Saro H. Armenian, DO, MPH \\ Sharon M. Castellino, MD, MSc ${ }^{4,5}$; David C. Hodgson, MD, MPH'; and Melissa M. Hudson, MD ${ }^{1,2}$
}

\begin{abstract}
The successful integration of clinical trials into pediatric oncology has led to steady improvement in the 5-year survival rate for children diagnosed with Hodgkin lymphoma (HL). It is estimated that $>95 \%$ of children newly diagnosed with HL will become long-term survivors. Despite these successes, survival can come at a cost. Historically, long-term survivors of HL have a high risk of late-occurring adverse health effects and increased risk of nonrelapse mortality compared with the general population. The recognition of late-occurring events paired with the decades of life remaining for children cured of $\mathrm{HL}$ have made paramount the need to develop effective treatments that minimize the risk of late toxicity. Toward this goal, multiple, dose-intense, risk- and response-based regimens that use lower cumulative doses of chemotherapy and radiation have been developed. Appropriate frontline treatment selection requires a level of familiarity with the efficacy, acute toxicity, convenience, and late effects of treatments that may be impractical for providers who infrequently treat children with HL. There is an increasing need for guideline developers to begin to merge considerations from both frontline treatment and survivorship guidelines into practical documents that integrate potential long-term health risks. Herein, we take the first steps toward doing so by aligning cumulative treatment exposures, anticipated risks of late toxicity, and suggested surveillance recommendations for NCCN-endorsed Pediatric HL Guidelines. Future studies that integrate simulation modeling will strengthen this integrated approach and allow for opportunities to incorporate regimen-specific risks, health-related quality of life, and cost-effectiveness into decision tools to optimize HL therapy.
\end{abstract}

J Natl Compr Canc Netw 2021;19(6):755-764 doi: 10.6004/jnccn.2021.7042

${ }^{1}$ Department of Oncology, and ${ }^{2}$ Department of Epidemiology and Cancer Control, St. Jude Children's Research Hospital, Memphis, Tennessee; ${ }^{3}$ Departments of Pediatrics and Population Sciences, City of Hope

Comprehensive Cancer Center, Duarte, California; ${ }^{4}$ Department of Pediatrics, Hematology-Oncology, Emory University School of Medicine, Atlanta, Georgia; ${ }^{5}$ Aflac Cancer and Blood Disorders Center, Children's Healthcare of Atlanta, Atlanta, Georgia; and ${ }^{6}$ Department of Medicine, Princess Margaret Cancer Centre, Toronto, Ontario, Canada.
In the United States, approximately 1 in 78,500 children aged 0 to 19 years is diagnosed with Hodgkin lymphoma (HL) annually, accounting for an estimated 1,050 incident patients per year. ${ }^{1}$ Although the annual incidence per million population is $<5$ in children aged $<10$ years, it is 32.5 in adolescents aged 15 to 19 years, making HL the most common malignancy occurring in this age group. ${ }^{1}$ Recognition that radiation alone could effectively treat HL resulted in cures even at the turn of the 20th century. ${ }^{2,3}$ Subsequently, the 5 -year survival rate for children aged $<20$ years at diagnosis has steadily improved, now exceeding 95\% (Figure 1). ${ }^{1}$ Despite these successes, survival comes at the cost of increased late morbidity ${ }^{4-7}$ and mortality compared with the general population. ${ }^{7,8}$

\section{Progress Over Time and Divergence of Pediatric From Adult Regimens}

Successful pediatric HL clinical trials have improved survival rates and reduced short- and long-term toxicity, resulting in an increasing number of long-term survivors. Although overall survival rates with contemporary therapy in children and adolescents are exceptional, subgroups experience suboptimal initial disease-free survival. ${ }^{9} \mathrm{Al}-$ though historical definitions of risk have varied, 5-year disease-free survival rates generally range from $80 \%$ to $97 \%$ for low-risk disease and from $77 \%$ to $90 \%$ for highrisk disease. ${ }^{9}$ Stages IIB with bulk (pediatric), IIIB, and IVB disease have been uniformly considered as requiring high-risk treatment, whereas stage IIIA disease has been variably classified as either intermediate- or high-risk in pediatric trials, as have stages IIB and IVA disease in adult trials. Over time, risk stratification has evolved beyond its early dependence on Ann Arbor stage alone to now include bulky and extranodal lesions as higher-risk disease characteristics; however, both have been inconsistently included in high-risk groups in clinical trials. ${ }^{10}$

Risk-stratified treatment approaches, partially motivated by dose-dependent associations between specific treatment exposures and late morbidity and mortality, ${ }^{7}$ have reduced treatment-related organ toxicity and subsequent neoplasms in survivors of pediatric HL. ${ }^{10}$ For 


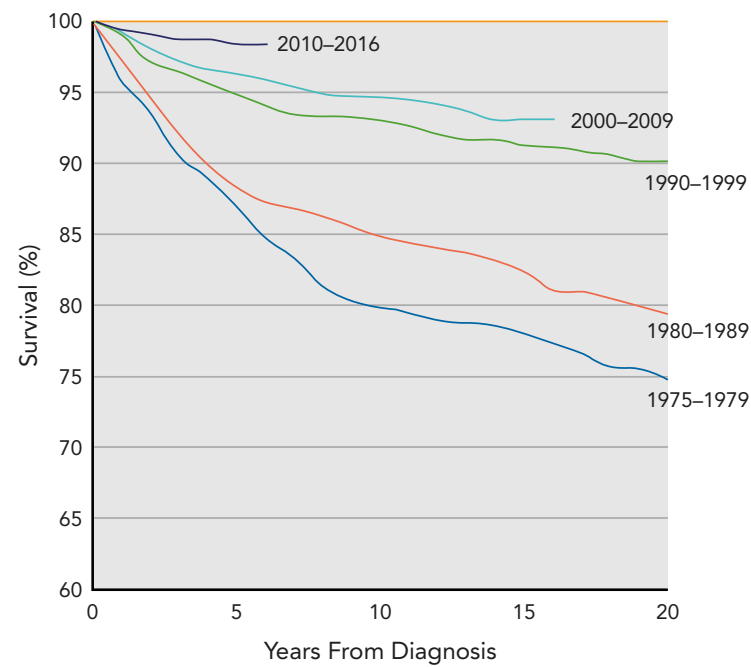

Figure 1. Overall survival rates for pediatric Hodgkin lymphoma by decade. ${ }^{1}$

example, recognition that young women exposed to mantle radiation were at high risk of subsequent breast cancer motivated the tailoring of radiotherapy, with respect to both overall use and tissue volume exposed. ${ }^{6,11}$ In response, some adult regimens have used chemotherapy-only approaches to avoid radiation, whereas pediatric protocols have relied on combined-modality therapy incorporating involved-field, low-dose radiation to simultaneously reduce the risk of late cardiac events. ${ }^{12-14}$ Recognition that high cumulative alkylating agent doses and gonadal radiation adversely affect both male and female reproductive function, ${ }^{15-17}$ and knowledge of the particularly deleterious effects of procarbazine in male patients, ${ }^{18-20}$ prompted the development of alternative regimens that preserve cure rates without compromising fertility for most patients. ${ }^{21}$ These objectives have been achieved through several key changes.

First, the availability of granulocyte colonystimulating factor facilitated the adoption of dose-dense, collectively myelosuppressive regimens incorporating additional agents while simultaneously allowing for the reduction of cumulative doses of individual agents within regimens. ${ }^{22,23}$ For example, the incorporation of etoposide into pediatric regimens enhanced antitumor efficacy while reducing cumulative alkylating agent doses. ${ }^{22-26}$ Together, these approaches allowed the containment of cumulative doxorubicin-equivalent doses of anthracyclines $^{27,28}$ to $\leq 250 \mathrm{mg} / \mathrm{m}^{2}$ and cyclophosphamideequivalent doses of alkylating agents ${ }^{17}$ to $\leq 7 \mathrm{~g} / \mathrm{m}^{2}$.21,29,30 Such changes come with potential tradeoffs. For example, frequent scheduling and higher doses of etoposide have been associated with an increased risk of subsequent leukemia, ${ }^{31,32}$ although with contemporary pediatric $\mathrm{HL}$ regimens this risk is exceedingly low. ${ }^{10,33} \mathrm{In}$ addition, in adolescents with HL aged 17 to 21 years, treatment using an adult versus a pediatric approach resulted in approximately $10 \%$ fewer patients receiving radiation but an approximately $10 \%$ reduction in progression-free survival. ${ }^{34}$ Although this effect may be multifactorial, the implications of additional relapsed therapy must be considered.

Improved CT imaging and the emergence of FDGPET as a biomarker of chemotherapy response, combined with the aforementioned progress in qualifying baseline disease risk, led to the current risk-based, response-adapted treatment approach that has facilitated the reduction and elimination of consolidative radiation exposure for those achieving rapid resolution of disease activity. ${ }^{22,23}$ For individuals less responsive to chemotherapy, improvements in image-guided treatment and the transition from extended-field (eg, mantle) to involved-field radiation, and more recently involved-site or involved-node radiation, significantly reduced doses to normal tissues ${ }^{35}$ and contributed to clear reductions in posttreatment morbidity and premature mortality, ${ }^{36,37}$ most notably regarding subsequent neoplasms. ${ }^{38}$

\section{Need for Guidelines in Treatment of De Novo Pediatric HL}

Although successful cooperative group HL trials have yielded several efficacious treatment strategies, heterogeneity in risk stratification and response criteria has made it challenging to identify a "single-best" approach in pediatric patients. ${ }^{10}$ Now more than ever, balancing cure and long-term toxicity, risks and benefits of long-term surveillance, and increasing recognition of the importance of the quality and not just the quantity of life-years has challenged treatment decision-making. Thus, the development of the pediatric-specific NCCN Clinical Practice Guidelines in Oncology (NCCN Guidelines) for HL is a timely, much-needed step to facilitate progress toward personalized treatment. ${ }^{39}$

Comparisons between cumulative exposures for regimens endorsed by both the pediatric and adult classic HL NCCN Guidelines can be found in Tables 1-3. Differences in cumulative exposures are largely determined by disease risk stratification at diagnosis, early response to therapy, number of treatment cycles, and need for consolidative radiation as defined by the protocol (eg, radiation to large mediastinal adenopathy regardless of response when treating high-risk disease using doxorubicin/bleomycin/vincristine/etoposide/ prednisone/cyclophosphamide (ABVE-PC). Because a limited number of cycles are required to cure low-risk HL, the cumulative dose differences and thus the coinciding late health risks are minimal between endorsed regimens in low-risk individuals who achieve an 
adequate early response to therapy. However, differences emerge for those who do and do not require additional consolidative chemotherapy or radiation. For example, when treating low-risk HL using doxorubicin/ vincristine/prednisone/cyclophosphamide (AVPC) or vincristine/etoposide/prednisone/doxorubicin (OEPA), a higher risk of late effects is directly related to organspecific radiation exposure in those achieving inadequate early responses to therapy because no additional consolidative chemotherapy is used. However, when beginning with doxorubicin/bleomycin/vinblastine/dacarbazine (ABVD), selection of the additional consolidative regimen can significantly impact the cumulative anthracycline exposure $\left(100-300 \mathrm{mg} / \mathrm{m}^{2}\right)$ regardless of the need for radiation based on response.

Similarly, the differences in late health risks become more apparent between the various intermediate- and high-risk regimens (Tables 1-3). For example, in the high-risk regimens, the cumulative doxorubicin exposure with 6 cycles of ABVD is $300 \mathrm{mg} / \mathrm{m}^{2}$ compared with $250 \mathrm{mg} / \mathrm{m}^{2}$ with 5 cycles of ABVE-PC and $160 \mathrm{mg} / \mathrm{m}^{2}$ with 6 total cycles of OEPA + cyclophosphamide/ vincristine/prednisone/dacarbazine (OEPA-COPDAC). In contrast, lower exposure to anthracyclines in the ABVE-PC and OEPA-COPDAC regimens comes at the expense of higher alkylating agent exposure that can be avoided with ABVD. In addition, 6 cycles of ABVD affords an intentional omission of radiation, whereas high-risk pediatric regimens use PET-only, responseadapted radiation in the absence of large mediastinal adenopathy (ie, with ABVE-PC), allowing for an omission of radiation in a large percentage of patients, highlighting the tradeoffs to consider when choosing the best regimen for individual patients.

Although the tolerability of acute adverse events (eg, reduced nausea/emesis, neuropathy, myelosuppression, and related fever and neutropenia) and the efficacy and convenience of outpatient biweekly delivery favor ABVD for adult oncology practices, the potential for late cardiotoxicity in children and adolescents due to higher cumulative doses of doxorubicin, magnified by concurrent chest-directed radiation with historical modalities and doses, has led to a historical divergence from this approach in pediatric oncology practices. ${ }^{10}$ In this regard, pediatric regimens have distinct advantages in young patients; however, they have also introduced new exposures with unique acute and late toxicity risk profiles (eg, epipodophyllotoxins and risk of subsequent leukemia). In addition, they require consecutive day infusions, which are potentially challenging for working caregivers and adolescents/adults. As delineated in the NCCN Guidelines, ${ }^{39}$ it is critical to weigh the risks and benefits of both the acute and late toxicity profiles of each treatment regimen for each patient.

\section{Rigor in Guideline Development and Evaluation of Late Effects Screening}

It is important to reiterate that many late effects will not become clinically apparent until decades after cancer treatment. In adolescent and young adult populations transitioning from pediatric to adult practices, this latency raises concern regarding continuing screening and recognition of adverse health events, potentially leading to poorer outcomes. Awareness and anticipation of potential cancer treatment-related late effects by survivors and providers are essential to facilitate timely interventions to prevent or decrease the burden of health complications and optimize quality of life. Clinical practice guidelines exist to inform screening in childhood cancer survivors. ${ }^{40,41}$ In North America, the Children's Oncology Group guidelines are frequently used and combine evidence linking therapeutic exposures to specific late effects, with screening recommendations aligning the magnitude of risk with screening intensity. ${ }^{41}$

In the general medical community, increasing awareness regarding the adverse effects of intensive lifelong screening ${ }^{42}$ has led to "value-based" consideration of the tradeoffs between the benefits, harms, and costs of screening strategies. ${ }^{42}$ High-value strategies provide a quantifiable benefit that clearly justifies the harms and associated costs, whereas low-value strategies result in disproportionately small health benefits relative to the harms and costs incurred. ${ }^{42}$ Value and intensity are not the same, ${ }^{42,43}$ however, and although more intensive screening may provide greater benefit in lives extended, it can result in higher levels of false-positive results, diagnostic workups, overdiagnosis, costs, and patient anxiety that may not be justified by the incremental increase in benefit. ${ }^{42,44,45}$ For example, although data support screening for survivors of childhood cancer at high risk of breast cancer due to chest radiation, ${ }^{46}$ the false-positive rates in women at average risk in the general population are not insignificant. ${ }^{47,48}$ Optimizing value therefore requires finding the intermediate level of intensity that best balances benefits with harms and costs. ${ }^{42}$

There are inherent challenges to conducting research optimizing value-based screening in survivors of childhood cancer, including the long latency to many late effects, the large numbers needed to detect a significant effect, the evolution of treatment approaches, and the lack of clinical equipoise. ${ }^{49}$ Screening intensity has thus historically relied on expert opinion to determine the appropriateness of recommendations. To overcome these challenges, researchers have used simulation modeling to evaluate the clinical effectiveness and cost-effectiveness of endorsed strategies. ${ }^{46,49-51}$ Despite being limited in scope (radiation-related breast cancer, ${ }^{46,52,53}$ colorectal cancer, ${ }^{54}$ anthracycline-related cardiomyopathy ${ }^{49-51}$ ), these efforts have facilitated the refinement and often 


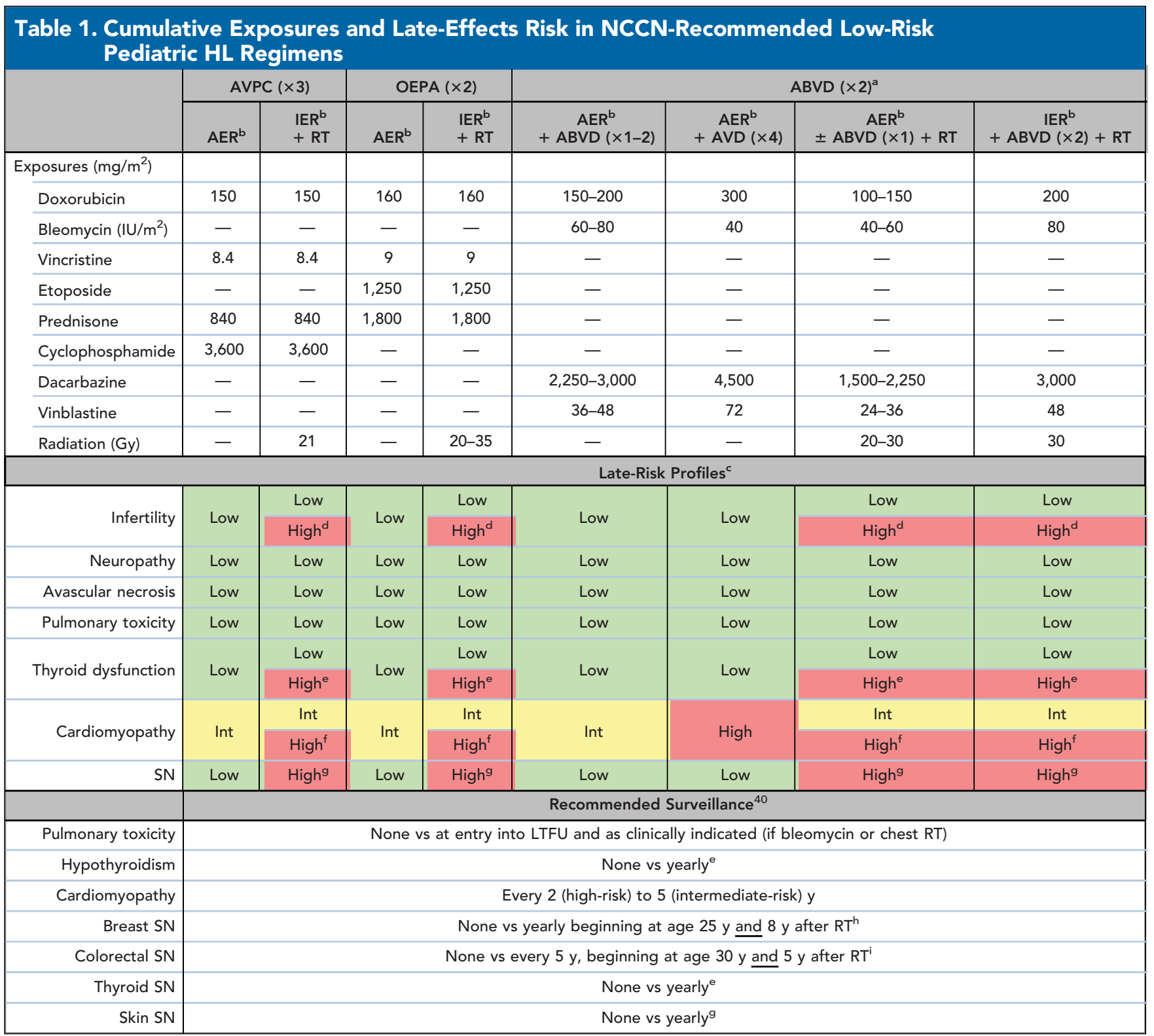

Abbreviations: ABVD, doxorubicin/bleomycin/vinblastine/dacarbazine; AER, adequate early response; AVD, doxorubicin/vinblastine/dacarbazine; AVPC doxorubicin/vincristine/prednisone/cyclophosphamide; BEACOPP, bleomycin/etoposide/doxorubicin/cyclophosphamide/vincristine/procarbazine/prednisone; $\mathrm{HL}$, Hodgkin lymphoma; IER, inadequate early response; Int, intermediate; LTFU, long-term follow-up; RT, radiation therapy; SN, subsequent neoplasm. ${ }^{a}$ Ranges reflect strategies outlined in the NCCN Clinical Practice Guidelines in Oncology: Hodgkin Lymphoma, Version 2.2021 (age $\geq 18$ years; available at NCCN.org).

${ }^{b}$ Response assessments based upon FDG-PET/CT, PET/MRI, and/or contrast-enhanced diagnostic CT or MRI of original sites of disease as per primary study methodology.

${ }^{c}$ Risk reflects published guideline stratification for infertility ${ }^{61}$ and cardiomyopathy. ${ }^{62}$ Neuropathy, avascular necrosis, and pulmonary toxicity were considered low if incidence in clinical trials was $<5 \%$ and intermediate if $\geq 5 \% .{ }^{23,25,31,63-65}$ Thyroid dysfunction risk was considered high if mean thyroid RT dose was $>10 \mathrm{~Gy}$ and otherwise was considered low. ${ }^{66} \mathrm{SN}$ risk was considered high depending on dose and volume of RT and otherwise was considered low. $\mathrm{d}_{\text {If }} \geq 4 \mathrm{~Gy}$ to testes or $\geq 15 \mathrm{~Gy}$ to both ovaries. ${ }^{61}$

e If mean thyroid RT dose was $>10 \mathrm{~Gy} .{ }^{67}$

${ }^{f}$ If $\geq 15$ Gy chest RT. ${ }^{62}$

${ }^{9}$ Depending on RT dose and volume. ${ }^{40}$

${ }^{\text {h} A n y ~ b r e a s t ~ R T . ~}{ }^{40,68}$

iAny abdomen/pelvis RT. ${ }^{40,69}$

the deintensification of recommended screening frequencies. ${ }^{46,49-51}$ Notably, they have paved the way for much-needed efforts to evaluate existing screening recommendations across a broader set of health outcomes and to assess multilevel interventions seeking to reduce the burden of morbidity in survivors. 


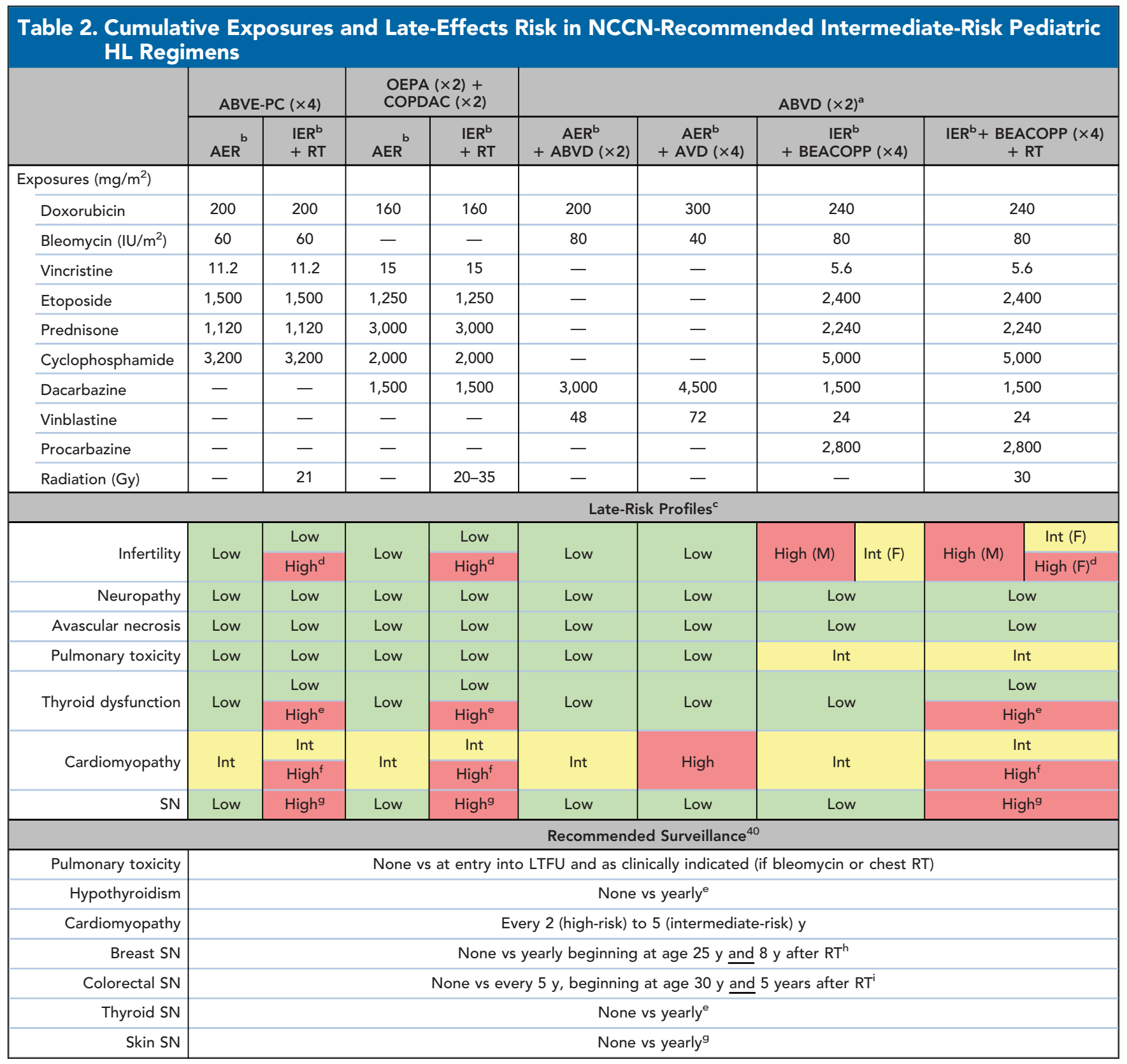

Abbreviations: ABVD, doxorubicin/bleomycin/vinblastine/dacarbazine; ABVE-PC, doxorubicin/bleomycin/vincristine/etoposide/prednisone/cyclophosphamide AER, adequate early response; AVD, doxorubicin/vinblastine/dacarbazine; BEACOPP, bleomycin/etoposide/doxorubicin/cyclophosphamide/vincristine/procarbazine/prednisone; COPDAC, cyclophosphamide/vincristine/prednisone/dacarbazine; F, female; HL, Hodgkin lymphoma; IER, inadequate early response; Int, intermediate; LTFU, long-term follow-up; M, male; OEPA, vincristine/etoposide/prednisone/doxorubicin; RT, radiation therapy; SN, subsequent neoplasm.

${ }^{\text {a}}$ Ranges reflect strategies outlined in the NCCN Clinical Practice Guidelines in Oncology: Hodgkin Lymphoma, Version 2.2021 (age $\geq 18$ years; available at NCCN.org).

${ }^{\mathrm{b}}$ Response assessments based upon FDG-PET/CT, PET/MRI, and/or contrast-enhanced diagnostic CT or MRI of original sites of disease as per primary study methodology.

${ }^{c}$ Risk reflects published guideline stratification for infertility ${ }^{61}$ and cardiomyopathy. ${ }^{62}$ Neuropathy, avascular necrosis, and pulmonary toxicity were considered low if

incidence on clinical trials was $<5 \%$ and intermediate if $\geq 5 \% .{ }^{23,25,31,63-65}$ Thyroid dysfunction risk was considered high if mean thyroid RT dose was $>10$ Gy and otherwise was considered low. ${ }^{66} \mathrm{SN}$ risk was considered high depending on dose and volume of RT and otherwise was considered low.

${ }^{d}$ If $\geq 4$ Gy to testes or $\geq 15$ Gy to both ovaries. ${ }^{61}$

eIf mean thyroid RT dose was $>10 \mathrm{~Gy} .{ }^{67}$

${ }^{f}$ If $\geq 15$ Gy chest RT. ${ }^{62}$

${ }^{9}$ Depending on RT dose and volume. ${ }^{40}$

${ }^{\mathrm{h}}$ Any breast $\mathrm{RT} .{ }^{40,68}$

'Any abdomen/pelvis RT. ${ }^{40,69}$ 


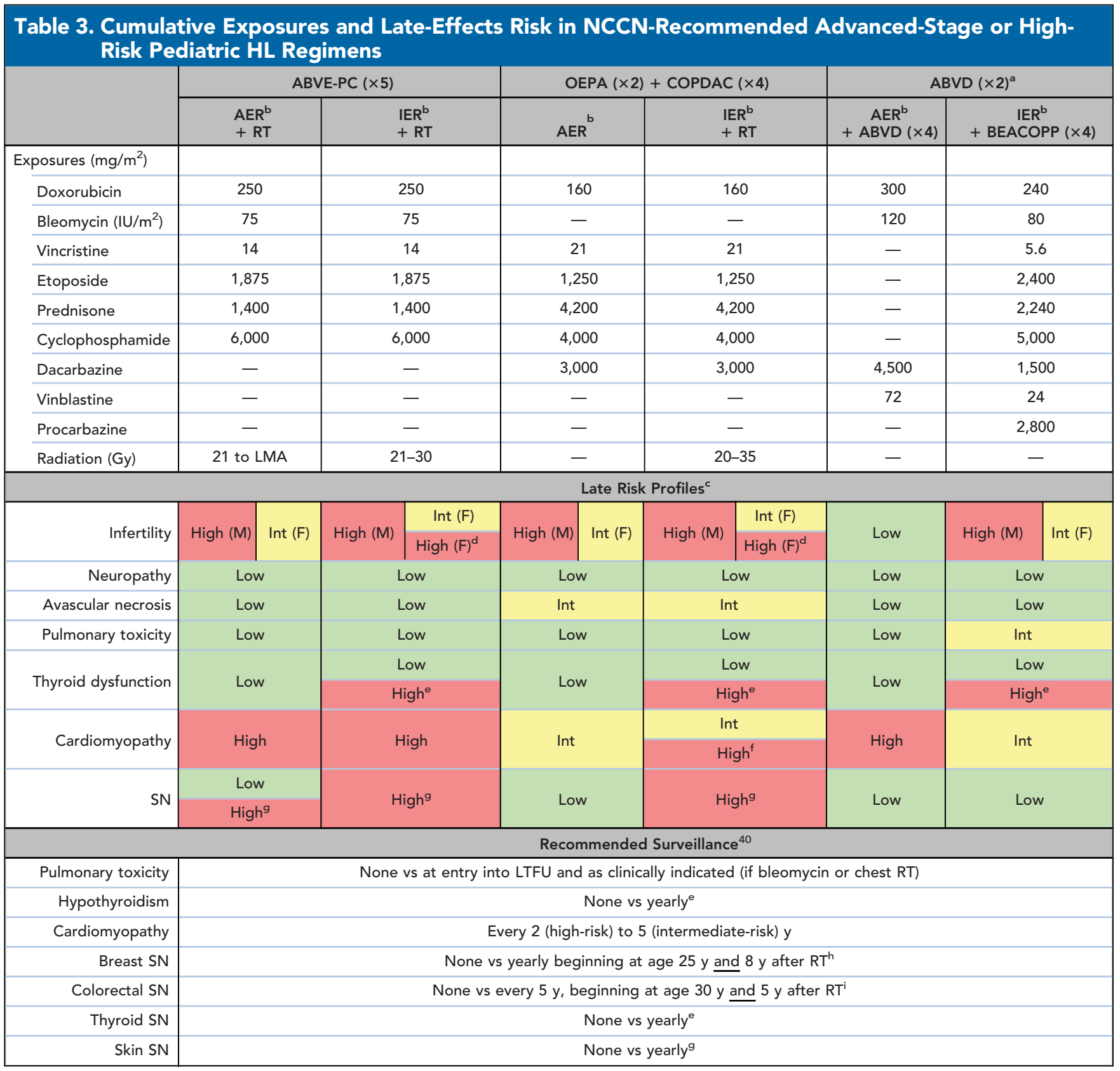

Abbreviations: ABVD, doxorubicin/bleomycin/vinblastine/dacarbazine; ABVE-PC, doxorubicin/bleomycin/vincristine/etoposide/prednisone/cyclophosphamide; AER, adequate early response; COPDAC, cyclophosphamide/vincristine/prednisone/dacarbazine; $F$, female; $H L$, Hodgkin lymphoma; IER, inadequate early response; Int, intermediate; LMA, large mediastinal adenopathy; LTFU, long-term follow-up; M, male; OEPA, vincristine/etoposide/prednisone/doxorubicin; RT, radiation therapy; SN, subsequent neoplasm.

${ }^{a}$ Ranges reflect strategies outlined in the NCCN Clinical Practice Guidelines in Oncology: Hodgkin Lymphoma, Version 2.2021 (age $\geq 18$ years; available at NCCN.org).

${ }^{\mathrm{b}}$ Response assessments based upon FDG-PET/CT, PET/MRI, and/or contrast-enhanced diagnostic CT or MRI of original sites of disease as per primary study methodology.

${ }^{c}$ Risk reflects published guideline stratification for infertility ${ }^{61}$ and cardiomyopathy. ${ }^{62}$ Neuropathy, avascular necrosis, and pulmonary toxicity were considered low if incidence on clinical trials was $<5 \%$ and intermediate if $\geq 5 \% .{ }^{23,25,31,63-65}$ Thyroid dysfunction risk was considered high if mean thyroid RT dose was

$>10 \mathrm{~Gy}$ and otherwise was considered low. ${ }^{66} \mathrm{SN}$ risk was considered high depending on dose and volume of RT and otherwise was considered low.

${ }^{d}$ If $\geq 4$ Gy to testes or $\geq 15$ Gy to both ovaries. ${ }^{61}$

eIf mean thyroid RT dose was $>10 \mathrm{~Gy} .{ }^{67}$

$f_{\text {If }} \geq 15$ Gy chest RT. ${ }^{62}$

${ }^{9}$ Depending on RT dose and volume. ${ }^{40}$

${ }^{\text {h}}$ Any breast RT. ${ }^{40,68}$

'Any abdomen/pelvis RT. ${ }^{40,69}$ 


\section{Integration of Frontline Treatment and Late Effects Guidelines}

The selection of NCCN-preferred regimens for de novo, pediatric, classic HL largely reflects efficacy and provider familiarity. However, providers, in combination with patients and caregivers, must also consider the risks of long-term health consequences when selecting the optimal regimen for individual patients. Existing literature $^{4-8,55-57}$ and guidelines ${ }^{40,41}$ should be simultaneously considered before and after the selection of therapy for each patient. As previously mentioned, late effects guidelines outline potential health consequences and recommended surveillance strategies. ${ }^{40,41}$ However, parallel consideration of treatment selection based on efficacy, acute toxicity, convenience, and late effects requires a familiarity with both treatment and follow-up guidelines that is impractical for providers who infrequently treat children with HL. There is therefore an increasing need for guideline developers to merge considerations from both frontline treatment and survivorship guidelines into practical documents that integrate potential long-term health risks. Tables 1 to 3 represent initial steps toward this objective by characterizing NCCN-endorsed treatment regimens by cumulative exposure, risk for associated late effects, and impact on subsequent screening recommendations. We assigned risk based on published guidelines where available (ie, gonadal toxicity ${ }^{58}$ and cardiomyopathy ${ }^{59}$ ). For conditions in which dose-dependent associations with late toxicity were unavailable (eg, neuropathy, pulmonary toxicity, avascular necrosis), we relied on reported incidences from clinical trials, because these particular conditions largely represent long-term (ie, occurring during therapy and persisting long-term) rather than late-occurring conditions. Conditions occurring in approximately $<5 \%$ of study patients were considered low-risk, and those occurring in $5 \%$ to $10 \%$ of patients were considered intermediaterisk. We did not identify more frequently occurring

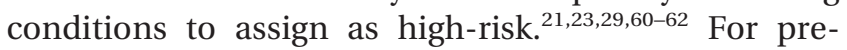
dominantly radiation-dependent conditions (ie, thyroid dysfunction ${ }^{63}$ and subsequent neoplasms), ${ }^{64-66}$ risk was considered high or low based on receipt or no receipt of prior radiation.

Largely because of the aforementioned successes in treatment and cure, pediatric HL represents a unique population in which to pioneer the convergence of frontline treatment and late-toxicity guidelines. Although our efforts reflect well-described associations between individual treatment exposures and specific outcomes, they represent the relative risk of events taken from individual trials and cohorts. Risk derived from clinical trial reports to date may underestimate low-grade conditions, including fatigue and neuropathy, that often go unreported in such studies yet may still have a significant impact on functional outcomes or quality of life. It is difficult to compare the impact of "risk" for different adverse events on decision-making without better understanding patient perspectives. For example, although occurrences of treatment-related leukemia associated with HL regimens are infrequent, its devastating implications may influence some patients to accept a higher incidence of other toxicities instead. Moreover, risk of late toxicity is substantially greater for individuals who experience relapse and receive additional cytotoxic chemotherapy, new or extended-field radiation exposure, and/or hematopoietic cell transplant. It is therefore important to balance these considerations with new trial development and in the selection of endorsed frontline regimens.

Although they are consistent with existing guideline approaches, ${ }^{41}$ our risk estimates could be strengthened by a number of steps. First, investigations into protocolspecific late health risks reflecting combinations of exposures (eg, ABVE-PC as a collective treatment) are needed. These data, although lacking in HL trials, have been alternatively assessed in recently treated survivors of B-cell non-Hodgkin lymphoma. ${ }^{67}$ This strategy is dependent on the availability of large datasets containing uniformly treated cohorts of patients. Second, although many lateeffects studies have categorized radiation exposure by prescribed dose, it is increasingly well recognized that this is a crude indicator of organ-specific dose exposures, which are a more accurate indicator of late-toxicity risk. The Pediatric Normal Tissue Effects in the Clinic collaboration is working to create better quantitative tissue-specific dose-risk information necessary to refine guidelines for both treatment and follow-up. ${ }^{68}$ In addition, consideration of modifiable risks factors present at diagnosis could inform treatment selections. For example, hypertension has been shown to potentiate anthracycline-mediated risk for heart failure in survivors of childhood cancer. ${ }^{69}$ It is reasonable, therefore, to consider that given a choice between equally efficacious regimens, one may choose a lower anthracycline-containing regimen, or administration of a cardioprotective agent, for an obese patient with a strong family history of hypertension. It will also be critical to incorporate health status and health-related quality of life into future decisionmaking algorithms, including the modifying impact of interventions (eg, the impact of fertility preservation on how a patient weighs the risk of infertility). Equally important will be the consideration of not only tradeoffs between dichotomous exposures (eg, radiation vs no radiation) but also whether the addition of novel agents (eg, CD30-targeted therapy and PD-1 inhibitors), treatment reductions for patients who later experience relapse, and/or contemporary radiation modalities 
that mitigate exposure to healthy tissue (eg, proton therapy) will result in more acceptable collective toxicity profiles.

These strategies are difficult if not impossible to prospectively evaluate in randomized clinical trials given the short duration of follow-up relative to the long latency to incident late effects. Simulation models offer one avenue for estimating an individual's risk for a given late effect and can be integrated into shared decision-making. Parsons et $\mathrm{al}^{70}$ showed the promise of simulated algorithms to determine the risks associated with combination therapy and to understand and facilitate discussion of these late health risks at diagnosis. Integrating health utilities based on risk estimates from multiple datasets, they simulated differences in late health outcomes and qualityadjusted life expectancy between chemotherapy-only and combined-modality regimens. ${ }^{70}$ Similarly, Bhakta et $\mathrm{al}^{71}$ simulated life expectancy and quality of life for patients treated using either ABVD or OEPA-COPDAC. Although the latter requires validation, both studies offer exciting potential for decision-making tools that will facilitate personalized HL treatment strategies and will benefit from large-scale collaborations and pooling of individual patient-level data, which are emerging as critical resources for HL and other diseases. For example, the Hodgkin Lymphoma International Study for Individual Care dataset compiles data from numerous large pediatric and adult treatment HL trials and international datasets. ${ }^{70}$ From this pooled dataset, authors have begun to use simulation models to estimate projected life expectancy, quality-adjusted life expectancy, and cause of death for survivors of HL treated in contemporary trials. ${ }^{72}$ The Pediatric Cancer Data Commons is another pooled dataset under development that merges individual study data limited to pediatric HL trials. ${ }^{57}$ Both show the capabilities of large collaborative efforts and highlight the potential for such datasets to inform future treatment guidelines.

\section{Screening Implementation}

Notably, $>80 \%$ of long-term survivors of childhood cancer are no longer followed by their treating institution and instead receive follow-up care in primary care settings. ${ }^{73}$ Although this transition is inevitable for all childhood cancers, it occurs sooner in survivors of HL given that many are diagnosed in adolescence and have fewer years remaining within pediatric practices, potentially complicating surveillance adherence. Several care delivery models ${ }^{74}$ have been used to facilitate provider and survivor awareness of and adherence to surveillance recommendations in primary care settings, in addition to providing survivorship care plans. ${ }^{75}$ Despite recent evidence of improvement, poor adherence still impedes optimal survivorship care delivery, ${ }^{76}$ highlighting a need to explore novel mechanisms, such as the use of motivational interviewing ${ }^{77,78}$ and mHealth, ${ }^{79,80}$ to engage long-term survivors and providers in routine survivorship care. In the interim, the implementation of models of care best fit to local resources, and open and clear communication between providers are essential to optimizing survivorship care delivery.

\section{Summary}

Collaboration through cooperative group trials and longterm follow-up cohort studies has resulted in multiple effective pediatric HL treatment options. Although all are reasonable, late-effects profiles differ between risk groups and regimen. We have integrated cumulative dose, lateeffects risk, and long-term follow-up surveillance recommendations with NCCN Guidelines-endorsed pediatric treatment regimens ${ }^{39}$ to facilitate shared decisionmaking between providers and patients. Future studies (eg, simulation models) will strengthen this integrated approach and allow opportunities to incorporate regimen-specific risks, health-related quality of life, and clinical effectiveness and cost-effectiveness into decision-making tools seeking to better personalize HL treatment selections.

Submitted January 19, 2021; revision received March 31, 2021; accepted for publication April 5, 2021.

Disclosures: The authors have disclosed that they have no financial interests, arrangements, or affiliations with the manufacturers of any products discussed in this article or their competitors.

Correspondence: Matthew J. Ehrhardt, MD, MS, Departments of Oncology and Epidemiology and Cancer Control, St. Jude Children's Research Hospital, 262 Danny Thomas Place, MS-735, Memphis, TN 38105. Email: matthew.ehrhardt@stjude.org

\section{References}

1. Howlader N, Noone AM, Krapcho M, et al. SEER Cancer Statistics Review (CSR) 1975-2017. Accessed April 13, 2021. Available at: https://seer. cancer.gov/csr/1975_2017/

2. Pusey WA. Cases of sarcoma and of Hodgkin's disease treated by exposures to X-rays-a preliminary report. JAMA 1902;XXXVIII:166-169.

3. Gilbert R. Notre méthode de roentgenthérapie de la lymphomagranulomatose (Hodgkin): résultats éloignés. Acta Radiol 1931;12:523-529.

4. Bhuller KS, Zhang Y, Li D, et al. Late mortality, secondary malignancy and hospitalisation in teenage and young adult survivors of Hodgkin lymphoma: report of the Childhood/Adolescent/Young Adult Cancer Survivors Research Program and the BC Cancer Agency Centre for Lymphoid Cancer. Br J Haematol 2016;172:757-768.

5. Hodgson DC, Gilbert ES, Dores GM, et al. Long-term solid cancer risk among 5-year survivors of Hodgkin's lymphoma. J Clin Oncol 2007;25:1489-1497.

6. Bhatia S, Robison LL, Oberlin O, et al. Breast cancer and other second neoplasms after childhood Hodgkin's disease. N Engl J Med 1996;334:745-751. 
7. Castellino SM, Geiger AM, Mertens AC, et al. Morbidity and mortality in long-term survivors of Hodgkin lymphoma: a report from the Childhood Cancer Survivor Study. Blood 2011;117:1806-1816.

8. de Vries S, Schaapveld M, Janus CPM, et al. Long-term cause-specific mortality in Hodgkin lymphoma patients [published online December 22, 2020]. J Natl Cancer Inst, doi: 10.1093/jnci/djaa194

9. Kelly KM. Hodgkin lymphoma in children and adolescents: improving the therapeutic index. Blood 2015;126:2452-2458.

10. Mauz-Körholz C, Metzger ML, Kelly KM, et al. Pediatric Hodgkin lymphoma. J Clin Oncol 2015;33:2975-2985.

11. Moskowitz CS, Chou JF, Wolden SL, et al. Breast cancer after chest radiation therapy for childhood cancer. J Clin Oncol 2014;32:2217-2223.

12. Bhakta N, Liu Q, Yeo F, et al. Cumulative burden of cardiovascular morbidity in paediatric, adolescent, and young adult survivors of Hodgkin's lymphoma: an analysis from the St. Jude Lifetime Cohort Study. Lancet Oncol 2016;17:1325-1334.

13. Aleman BM, van den Belt-Dusebout AW, De Bruin ML, et al. Late cardiotoxicity after treatment for Hodgkin lymphoma. Blood 2007;109:1878-1886.

14. Hahn $\mathrm{E}$, Jiang $\mathrm{H}, \mathrm{Ng} \mathrm{A}$, et al. Late cardiac toxicity after mediastinal radiation therapy for Hodgkin lymphoma: contributions of coronary artery and whole heart dose-volume variables to risk prediction. Int J Radiat Oncol Biol Phys 2017;98:1116-1123.

15. Chow EJ, Stratton KL, Leisenring WM, et al. Pregnancy after chemotherapy in male and female survivors of childhood cancer treated between 1970 and 1999: a report from the Childhood Cancer Survivor Study cohort. Lancet Oncol 2016;17:567-576.

16. Green DM, Liu W, Kutteh WH, et al. Cumulative alkylating agent exposure and semen parameters in adult survivors of childhood cancer: a report from the St Jude Lifetime Cohort Study. Lancet Oncol 2014;15:1215-1223.

17. Green DM, Nolan VG, Goodman PJ, et al. The cyclophosphamide equivalent dose as an approach for quantifying alkylating agent exposure: a report from the Childhood Cancer Survivor Study. Pediatr Blood Cancer 2014;61:53-67.

18. Schellong G, Pötter R, Brämswig J, et al. High cure rates and reduced long-term toxicity in pediatric Hodgkin's disease: the German-Austrian multicenter trial DAL-HD-90. J Clin Oncol 1999;17:3736-3744.

19. Chapman RM, Sutcliffe SB, Rees LH, et al. Cyclical combination chemotherapy and gonadal function. Retrospective study in males. Lancet $1979 ; 1: 285-289$

20. Whitehead E, Shalet SM, Blackledge G, et al. The effects of Hodgkin's disease and combination chemotherapy on gonadal function in the adult male. Cancer 1982;49:418-422.

21. Mauz-Körholz C, Hasenclever D, Dörffel W, et al. Procarbazine-free OEPA-COPDAC chemotherapy in boys and standard OPPA-COPP in girls have comparable effectiveness in pediatric Hodgkin's lymphoma: the GPOH-HD-2002 study. J Clin Oncol 2010;28:3680-3686.

22. Dörffel W, Lüders $H, R u ̈ h l ~ U$, et al. Preliminary results of the multicenter trial GPOH-HD 95 for the treatment of Hodgkin's disease in children and adolescents: analysis and outlook. Klin Padiatr 2003;215:139-145.

23. Schwartz CL, Constine LS, Villaluna D, et al. A risk-adapted, responsebased approach using ABVE-PC for children and adolescents with intermediate- and high-risk Hodgkin lymphoma: the results of P9425. Blood 2009;114:2051-2059

24. Friedmann AM, Hudson MM, Weinstein HJ, et al. Treatment of unfavorable childhood Hodgkin's disease with VEPA and low-dose, involvedfield radiation. J Clin Oncol 2002;20:3088-3094.

25. Landman-Parker J, Pacquement H, Leblanc T, et al. Localized childhood Hodgkin's disease: response-adapted chemotherapy with etoposide, bleomycin, vinblastine, and prednisone before low-dose radiation therapy-results of the French Society of Pediatric Oncology Study MDH90. J Clin Oncol 2000;18:1500-1507.

26. Tebbi CK, Mendenhall N, London WB, et al. Treatment of stage I, IIA, IIIA1 pediatric Hodgkin disease with doxorubicin, bleomycin, vincristine and etoposide (DBVE) and radiation: a Pediatric Oncology Group (POG) study. Pediatr Blood Cancer 2006;46:198-202.

27. Feijen $E A$, Leisenring $W M$, Stratton $K L$, et al. Equivalence ratio for daunorubicin to doxorubicin in relation to late heart failure in survivors of childhood cancer. J Clin Oncol 2015;33:3774-3780.

28. Feijen EAM, Leisenring WM, Stratton $\mathrm{KL}$, et al. Derivation of anthracycline and anthraquinone equivalence ratios to doxorubicin for late-onset cardiotoxicity. JAMA Oncol 2019;5:864-871.

29. Friedman DL, Chen L, Wolden S, et al. Dose-intensive response-based chemotherapy and radiation therapy for children and adolescents with newly diagnosed intermediate-risk Hodgkin lymphoma: a report from the Children's Oncology Group Study AHOD0031. J Clin Oncol 2014;32:3651-3658.

30. Körholz D, Claviez A, Hasenclever D, et al. The concept of the GPOHHD 2003 therapy study for pediatric Hodgkin's disease: evolution in the tradition of the DAL/GPOH studies. Klin Padiatr 2004;216:150-156.

31. Kollmannsberger C, Beyer J, Droz JP, et al. Secondary leukemia following high cumulative doses of etoposide in patients treated for advanced germ cell tumors. J Clin Oncol 1998;16:3386-3391.

32. Pui $\mathrm{CH}$, Ribeiro RC, Hancock ML, et al. Acute myeloid leukemia in children treated with epipodophyllotoxins for acute lymphoblastic leukemia. N Engl J Med 1991;325:1682-1687.

33. Giulino-Roth L, Pei Q, Buxton A, et al. Subsequent malignant neoplasms among children with Hodgkin lymphoma: a report from the Children's Oncology Group. Blood 2021;137:1449-1456.

34. Henderson TO, Parsons SK, Wroblewski KE, et al. Outcomes in adolescents and young adults with Hodgkin lymphoma treated on US cooperative group protocols: an adult intergroup (E2496) and Children's Oncology Group (COG AHOD0031) comparative analysis. Cancer 2018;124:136-144.

35. Hodgson DC, Dieckmann K, Terezakis S, et al. Implementation of contemporary radiation therapy planning concepts for pediatric Hodgkin lymphoma: guidelines from the International Lymphoma Radiation Oncology Group. Pract Radiat Oncol 2015;5:85-92.

36. Armstrong GT, Yasui Y, Robison LL. Reduction in late mortality after childhood cancer. N Engl J Med 2016;375:290-292.

37. Gibson TM, Mostoufi-Moab S, Stratton KL, et al. Temporal patterns in the risk of chronic health conditions in survivors of childhood cancer diagnosed 1970-99: a report from the Childhood Cancer Survivor Study cohort. Lancet Oncol 2018;19:1590-1601.

38. Turcotte LM, Liu $Q$, Yasui $Y$, et al. Temporal trends in treatment and subsequent neoplasm risk among 5-year survivors of childhood cancer, 1970-2015. JAMA 2017;317:814-824.

39. Flerlage JE, Hiniker SM, Armenian S, et al. NCCN Clinical Practice Guidelines in Oncology: Pediatric Hodgkin Lymphoma. Version 2.2021. Accessed January 2, 2021. To view the most recent version, visit NCCN.org

40. Kremer LC, Mulder RL, Oeffinger KC, et al. A worldwide collaboration to harmonize guidelines for the long-term follow-up of childhood and young adult cancer survivors: a report from the International Late Effects of Childhood Cancer Guideline Harmonization Group. Pediatr Blood Cancer 2013;60:543-549.

41. Landier W, Bhatia S, Eshelman DA, et al. Development of risk-based guidelines for pediatric cancer survivors: the Children's Oncology Group Long-Term Follow-Up Guidelines from the Children's Oncology Group Late Effects Committee and Nursing Discipline. J Clin Oncol 2004;22:4979-4990.

42. Harris RP, Wilt TJ, Qaseem A. A value framework for cancer screening: advice for high-value care from the American College of Physicians. Ann Intern Med 2015;162:712-717.

43. Owens DK, Qaseem A, Chou R, et al. High-value, cost-conscious health care: concepts for clinicians to evaluate the benefits, harms, and costs of medical interventions. Ann Intern Med 2011;154:174-180.

44. Hubbard RA, Kerlikowske K, Flowers $\mathrm{Cl}$, et al. Cumulative probability of false-positive recall or biopsy recommendation after 10 years of screening mammography: a cohort study. Ann Intern Med 2011;155:481-492.

45. Morioka-Douglas N, Hillard PJ. No Papanicolaou tests in women younger than 21 years or after hysterectomy for benign disease. JAMA Intern Med 2013;173:855-856.

46. Yeh JM, Lowry KP, Schechter CB, et al. Clinical benefits, harms, and cost-effectiveness of breast cancer screening for survivors of childhood cancer treated with chest radiation: a comparative modeling study. Ann Intern Med 2020;173:331-341.

47. Canelo-Aybar C, Ferreira DS, Ballesteros M, et al. Benefits and harms of breast cancer mammography screening for women at average risk of breast cancer: a systematic review for the European Commission Initiative on Breast Cancer. J Med Screen 2021;25:969141321993866.

48. Myers ER, Moorman P, Gierisch JM, et al. Benefits and harms of breast cancer screening: a systematic review. JAMA 2015;314:1615-1634.

49. Ehrhardt MJ, Ward ZJ, Liu Q, et al. Cost-effectiveness of the International Late Effects of Childhood Cancer Guideline Harmonization Group screening guidelines to prevent heart failure in survivors of childhood cancer. J Clin Oncol 2020;38:3851-3862.

50. Wong FL, Bhatia S, Landier W, et al. Cost-effectiveness of the Children's Oncology Group long-term follow-up screening guidelines for childhood 
cancer survivors at risk for treatment-related heart failure. Ann Intern Med 2014;160:672-683.

51. Yeh JM, Nohria A, Diller L. Routine echocardiography screening for asymptomatic left ventricular dysfunction in childhood cancer survivors: a model-based estimation of the clinical and economic effects. Ann Intern Med 2014;160:661-671.

52. Furzer J, Tessier L, Hodgson D, et al. Cost-utility of early breast cancer surveillance in survivors of thoracic radiation-treated adolescent Hodgkin lymphoma. J Natl Cancer Inst 2020;112:63-70.

53. Hodgson DC, Cotton C, Crystal P, et al. Impact of early breast cancer screening on mortality among young survivors of childhood Hodgkin's lymphoma. J Natl Cancer Inst 2016;108:djw010.

54. Gini A, Meester RGS, Keshavarz H, et al. Cost-effectiveness of colonoscopy-based colorectal cancer screening in childhood cancer survivors. J Natl Cancer Inst 2019;111:1161-1169.

55. Armstrong GT, Liu Q, Yasui Y, et al. Late mortality among 5-year survivors of childhood cancer: a summary from the Childhood Cancer Survivor Study. J Clin Oncol 2009;27:2328-2338.

56. Bhakta N, Liu Q, Ness KK, et al. The cumulative burden of surviving childhood cancer: an initial report from the St Jude Lifetime Cohort Study (SJLIFE). Lancet 2017;390:2569-2582.

57. Oeffinger KC, Mertens AC, Sklar CA, et al. Chronic health conditions in adult survivors of childhood cancer. N Engl J Med 2006;355:1572-1582.

58. Meacham LR, Burns K, Orwig KE, et al. Standardizing risk assessment for treatment-related gonadal insufficiency and infertility in childhood adolescent and young adult cancer: the Pediatric Initiative Network Risk Stratification System. J Adolesc Young Adult Oncol 2020;9:662-666.

59. Armenian SH, Hudson MM, Mulder RL, et al. Recommendations for cardiomyopathy surveillance for survivors of childhood cancer: a report from the International Late Effects of Childhood Cancer Guideline Harmonization Group. Lancet Oncol 2015;16:e123-136.

60. Johnson P, Federico M, Kirkwood A, et al. Adapted treatment guided by interim PET-CT scan in advanced Hodgkin's lymphoma. N Engl J Med 2016;374:2419-2429.

61. Kelly KM, Sposto R, Hutchinson R, et al. BEACOPP chemotherapy is a highly effective regimen in children and adolescents with high-risk Hodgkin lymphoma: a report from the Children's Oncology Group. Blood 2011;117:2596-2603.

62. Metzger ML, Link MP, Billett $A L$, et al. Excellent outcome for pediatric patients with high-risk Hodgkin lymphoma treated with brentuximab vedotin and risk-adapted residual node radiation [published online April 7, 2021]. J Clin Oncol, doi: 10.1200/JCO.20.03286

63. Sklar C, Whitton J, Mertens A, et al. Abnormalities of the thyroid in survivors of Hodgkin's disease: data from the Childhood Cancer Survivor Study. J Clin Endocrinol Metab 2000;85:3227-3232.

64. Veiga LH, Holmberg E, Anderson $\mathrm{H}$, et al. Thyroid cancer after childhood exposure to external radiation: an updated pooled analysis of 12 studies. Radiat Res 2016;185:473-484.

65. Mulder RL, Hudson MM, Bhatia S, et al. Updated breast cancer surveillance recommendations for female survivors of childhood, adolescent, and young adult cancer from the International Guideline Harmonization Group. J Clin Oncol 2020;38:4194-4207.
66. Teepen JC, de Vroom SL, van Leeuwen FE, et al. Risk of subsequent gastrointestinal cancer among childhood cancer survivors: a systematic review. Cancer Treat Rev 2016;43:92-103.

67. Ehrhardt MJ, Chen Y, Sandlund JT, et al. Late health outcomes after contemporary Lymphome Malin de Burkitt therapy for mature B-cell nonHodgkin lymphoma: a report from the Childhood Cancer Survivor Study. J Clin Oncol 2019:37:2556-2570.

68. Constine LS, Ronckers $\mathrm{CM}$, Hua $\mathrm{CH}$, et al. Pediatric Normal Tissue Effects in the Clinic (PENTEC): an international collaboration to analyse normal tissue radiation dose-volume response relationships for paediatric cancer patients. Clin Oncol (R Coll Radiol) 2019;31:199-207.

69. Armstrong GT, Oeffinger KC, Chen Y, et al. Modifiable risk factors and major cardiac events among adult survivors of childhood cancer. J Clin Oncol 2013;31:3673-3680.

70. Parsons SK, Kelly MJ, Cohen JT, et al. Early-stage Hodgkin lymphoma in the modern era: simulation modelling to delineate long-term patient outcomes. Br J Haematol 2018;182:212-221.

71. Bhakta N, Davies K, Turner J, et al. Impact of competing treatment scenarios on lifetime disease burden and quality adjusted life expectancy (QALE) among survivors of high-risk childhood Hodgkin lymphoma (HR-HL): a modeling study based on the St. Jude Lifetime (SJLIFE) cohort. Blood 2019;134(Suppl 1):267.

72. Parsons SK, Bhakta N, Rodday AM, et al. Lifelong disease burden of chemotherapy in Hodgkin lymphoma: a simulation study from the St. Jude Lifetime (SJLIFE) cohort and HL International Study for Individual Care (HoLISTIC) [abstract]. J Clin Oncol 2020;15(Suppl):Abstract 12068.

73. Oeffinger KC, Mertens AC, Hudson MM, et al. Health care of young adult survivors of childhood cancer: a report from the Childhood Cancer Survivor Study. Ann Fam Med 2004;2:61-70.

74. Oeffinger KC, McCabe MS. Models for delivering survivorship care. J Clin Oncol 2006:24:5117-5124.

75. Institute of Medicine and National Research Council; Hewitt M, Greenfield S, Stovall E, eds. From Cancer Patient to Cancer Survivor: Lost in Transition. Washington, DC: The National Academies Press; 2006.

76. Yan AP, Chen Y, Henderson TO, et al. Adherence to surveillance for second malignant neoplasms and cardiac dysfunction in childhood cancer survivors: a Childhood Cancer Survivor Study. J Clin Oncol 2020;38:1711-1722.

77. Hudson MM, Leisenring W, Stratton KK, et al. Increasing cardiomyopathy screening in at-risk adult survivors of pediatric malignancies: a randomized controlled trial. J Clin Oncol 2014;32:3974-3981.

78. Oeffinger KC, Ford JS, Moskowitz CS, et al. Promoting breast cancer surveillance: the EMPOWER study, a randomized clinical trial in the Childhood Cancer Survivor Study. J Clin Oncol 2019:37:2131-2140.

79. Armenian $\mathrm{SH}$, Lindenfeld L, lukuridze A, et al. Technology-enabled activation of skin cancer screening for hematopoietic cell transplantation survivors and their primary care providers (TEACH). BMC Cancer 2020;20:721

80. Daniel CL, Armstrong GT, Keske RR, et al. Advancing Survivors' Knowledge (ASK) about skin cancer study: study protocol for a randomized controlled trial. Trials 2015;16:109. 